ITM Web of Conferences 2, 01004 (2014)

DOI: $10.1051 /$ itmconf/20140201004

(C) Owned by the authors, published by EDP Sciences, 2014

\title{
From meso-scale to micro scale LES modelling: Application by a wake effect study for an offshore wind farm
}

\author{
M. Maché, H. Mouslim, and L. Mervoyer \\ INNOSEA, 1 rue de la Noë, CS 12102, 44321 Nantes, France
}

\begin{abstract}
The object of the study is to present the first step of the development of a methodology that aims to merge the gap between meso scale and micro scale modelling. WRF simulations have been carried out in order to consider global wind conditions over a meso scale area of the future wind farm. A statistical analysis of the global wind data over several years has been carried out to initiate the work for the Saint Nazaire site. A focus was made on two wind regimes: the most common wind typical of the site's location and the extreme winds over the period. The WRF results were then used as initial conditions for a micro scale simulation carried out by a non-stationary large-eddy simulation (SOWFA) tool based OpenFOAM solver that computes the flow around 2 turbines. Turbulence statistics were computed and analyzed. Fields of skewness and flatness show found that the distribution of turbulence becomes more homogeneous after the second turbine, while turbulence intensity increases.
\end{abstract}

\section{Introduction}

The construction of a new offshore wind farm has been awarded off the coast of Saint Nazaire in France within the country's first offshore wind tendering process. With $78 \mathrm{~km}^{2}$ surface and 80 wind turbines, the whole wind farm totals $480 \mathrm{MW}$ which should cover the power demand of 700000 inhabitants. The object of the work presented here is to go through a wind resource assessment for the wind farm area using numerical modelling which includes the effects of turbulent wind flow and wake effects as well as real local wind conditions.

Realistic onsite conditions were studied with the Weather Research \& Forecasting Model (WRF). It is worldwide widely used because it serves a large range of scales from thousands of kilometres to hundreds of meters and allows research applications as well as commercial ones. WRF is used for numerical weather prediction, assessment studies and aerodynamic studies with idealized conditions. The code is designed to run on massively parallel computers. Initially conceived in RANS mode, today, WRF disposes also a large-eddy-simulation model.

While in RANS simulations the impact of the turbulence on the mean flow is completely parameterized, LES filters and computes the large scales and models only small scales. The purpose of using LES is the realistic representation of turbulent flow. However inlet conditions coming from greater nest domains in RANS mode are not always compatible with LES domains. The lack of turbulence provides numeric problems in the entry of the LES simulation. [1] pointed out the importance of the

This is an Open Access article distributed under the terms of the Creative Commons Attribution License 2.0, which permits unrestricted use, distribution, and reproduction in any medium, provided the original work is properly cited. 


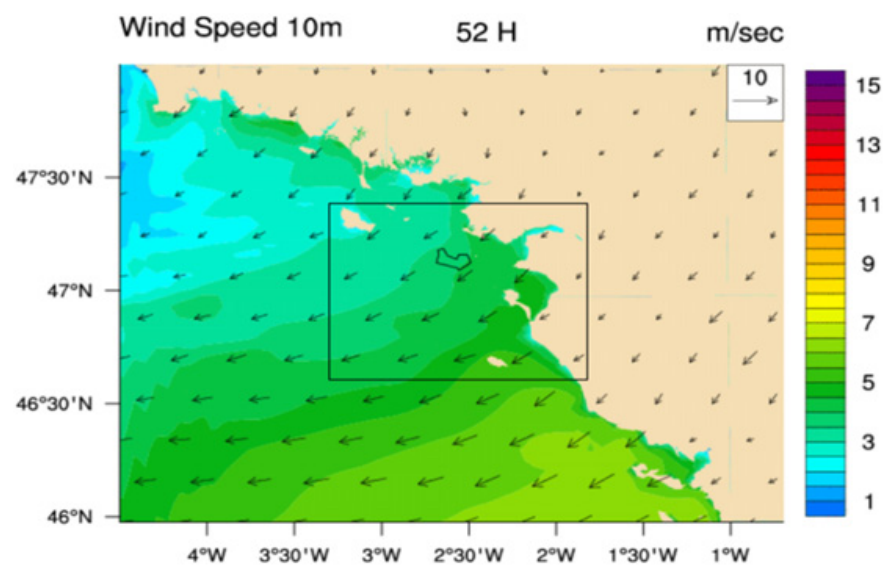

Figure 1. Example of meso-scale model wind speed output at $10 \mathrm{~m}$ around the future Saint Nazaire wind farm.

choice of the sub grid model and of a relaxing zone between the solution from the outer and the inner domain. Actually a grid nesting between a RANS model and a LES model takes place at a resolution that [2] called "Terra incognita": The spatio-temporal resolution is too high for an optimal utilization of a RANS model and not high enough for that of a LES model.

The LES is also a promising approach in wake modelling. The recent simulation tool SOWFA provides the wake effect of upwind turbines to the turbulent flow that arrives on the downwind turbine [3]. The wake effect, in addition to the effects of the atmospheric boundary layer turbulence provided by the LES approach, determines the production and the fatigue of the turbines within a wind farm.

This paper is the first step of a work that aims to merge the gap between meso scale modelling and micro scale modelling in the domain of wind energy. A case study is presented through a wind assessment of the future offshore wind farm at the site of Saint Nazaire. WRF-LES results from two days with different wind regimes, one typical and one extreme, were introduced as initial conditions in SOWFA. This solver based on OpenFOAM is used for the LES wind turbine simulations. In the latter, modelling the wake effect, from one turbine to its downwind neighbour, puts more light on the turbulence characteristics that can be transported from one turbine to another. Turbulence properties are then explored and commented.

\section{Meso-scale wind regime assessment}

\subsection{Purpose of the work}

In this section we study the wind characteristics of the Saint Nazaire wind farm region (latitude $47.16^{\circ}$, longitude $-2.6^{\circ}$ ) with the WRF-LES model: two typical wind regimes were chosen, a day representative for medium wind speed and a day representative for an extreme wind speed. First, a classic study with a WRF-RANS model of the Saint Nazaire region has been carried out. Eight years have been simulated according to the available measurement mast data nearby (1993 to 1995 and 2003 to 2007). We learn from this study that the average wind speed over the whole period is about $7 \mathrm{~m} / \mathrm{s}$ at $10 \mathrm{~m}$ and about $9 \mathrm{~m} / \mathrm{s}$ at $100 \mathrm{~m}$ from the ground. The most probable wind direction is $249^{\circ}$ where the wind blows from the ocean to the continent. This corresponds also to the most probable direction of extreme events. Monthly averages lie between 5 and $7 \mathrm{~m} / \mathrm{s}$ in summer months and between 7 and $9 \mathrm{~m} / \mathrm{s}$ in winter months. The map of the annual wind speed average shows that the region of the future wind farm of Saint Nazaire is located in the zone where statistically wind speed is maximal. Hence the position of the future wind 
Table 1. Relative and absolute error of the WRF results compared to mast measurements.

\begin{tabular}{|l|r|r|r|r|r|r|}
\hline \multirow{2}{*}{ Years } & \multicolumn{2}{|c|}{ Average speed } & \multicolumn{2}{c|}{ Max speed } & \multicolumn{2}{c|}{ Main direction } \\
\cline { 2 - 7 } & Abs (m/s) & Rel (\%) & Abs (m/s) & Rel $(\%)$ & Abs $\left({ }^{\circ}\right)$ & Rel (\%) \\
\hline $\mathbf{1 9 9 3}$ & 0.71 & 14.21 & 25.072 & 61.00 & -21 & -8.79 \\
\hline $\mathbf{1 9 9 4}$ & 0.60 & 12.34 & -0.463 & -3.01 & -2 & -0.80 \\
\hline $\mathbf{1 9 9 5}$ & 0.51 & 10.51 & 0.089 & 0.52 & -17 & -7.08 \\
\hline $\mathbf{2 0 0 3}$ & 0.11 & 2.73 & 0.28 & 1.77 & 20 & 7.17 \\
\hline $\mathbf{2 0 0 4}$ & 0.16 & 3.87 & 0.25 & 1.57 & 5 & 1.80 \\
\hline $\mathbf{2 0 0 5}$ & 0.18 & 4.50 & -1.14 & -7.67 & -5 & -1.82 \\
\hline $\mathbf{2 0 0 6}$ & 0.069 & 1.64 & 3.171 & 18.65 & -8 & -3.35 \\
\hline $\mathbf{2 0 0 7}$ & -0.09 & -2.18 & -2.265 & -15.73 & -12 & -4.53 \\
\hline
\end{tabular}

a)

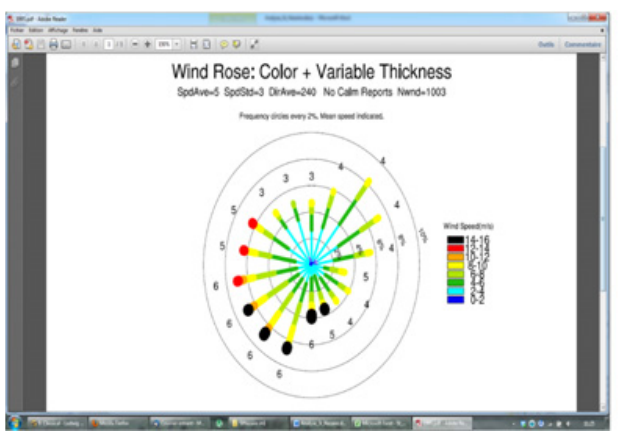

b)

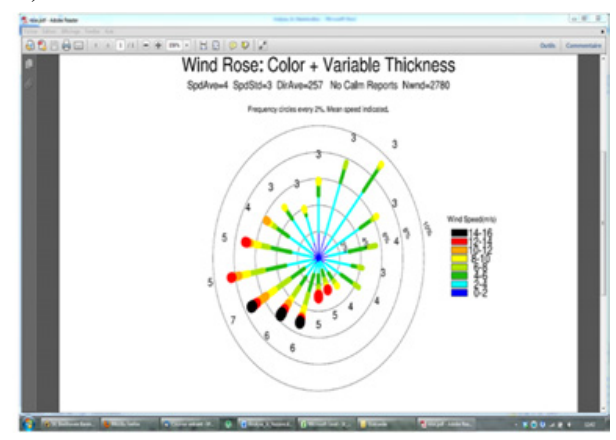

Figure 2. a) Wind rose for the whole year 1995 for the MeteoFrance onshore mast measurements, b) obtained by the WRF simulation.

farm combines a minimum coastal distance as it is close to shore and a maximum efficiency because it is still in a region where the land roughness effect is poor.

In order to validate the simulation, the results from WRF were compared to measurements of the meteorological mast of Saint Nazaire (Table 1). The wind roses of the year 1995 from WRF and from the measurements are compared in Figure 2. The relative occurrence of each sector differs less than 5\% between the two results.

From 1993 to 1995, the relative error of the average wind speed lies between 10 and $15 \%$. After 2003, the error in per cents for the average wind speed and main direction are inferior to $5 \%$. In fact, in 2003 the mast has been renewed and the quality of the measurements has increased. However the error percentages for the max wind speed is for both periods considerable.

Two main reasons can explain these discrepancies: Firstly WRF is forced every 6 hours from the NCEP $2.5^{\circ} \times 2.5^{\circ}$ data set that could be responsible for too smoothed wind speed, secondly, the temporal resolution of the WRF simulations could have been too coarse to capture extreme events.

\subsection{Methodology for wind regime selection}

Two wind regimes have been selected to run non-stationary LES calculations with SOWFA, one for average wind speed conditions and one for extreme wind speeds encountered at the aimed site. For these two periods, in order to improve the accuracy of these results, a nested simulation with the WRF-LES model has been performed. This simulation contained six domains. The resolution and the simulation mode are given in Table 2 and were adopted from [1] exactly in this form. For grid sizes of up to about $100 \mathrm{~m}$, LES mode is preferable [4]. Therefore the first three domains use the WRF-RANS 
Table 2. Principal simulation characteristics of the nested domains.

\begin{tabular}{|c|l|l|l|l|l|l|l|}
\hline Domain & Grid size (m) & Time Resolution (s) & Grid N-S & Grid O-E & Grid Z & Ratio & Mode \\
\hline D01 & 12150 & 72 & 82 & 91 & 109 & 1 & RANS \\
\hline D02 & 4050 & 24 & 82 & 91 & 109 & 3 & RANS \\
\hline D03 & 1350 & 8 & 82 & 91 & 109 & 3 & RANS \\
\hline D04 & 450 & $8 / 3$ & 82 & 91 & 109 & 3 & LES \\
\hline D05 & 150 & $8 / 9$ & 82 & 91 & 109 & 3 & LES \\
\hline D06 & 50 & $8 / 27$ & 100 & 100 & 109 & 3 & LES \\
\hline
\end{tabular}

a)

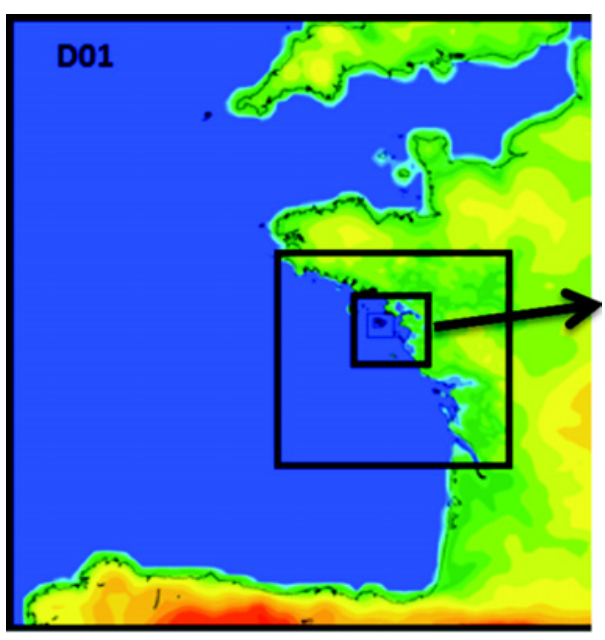

b)

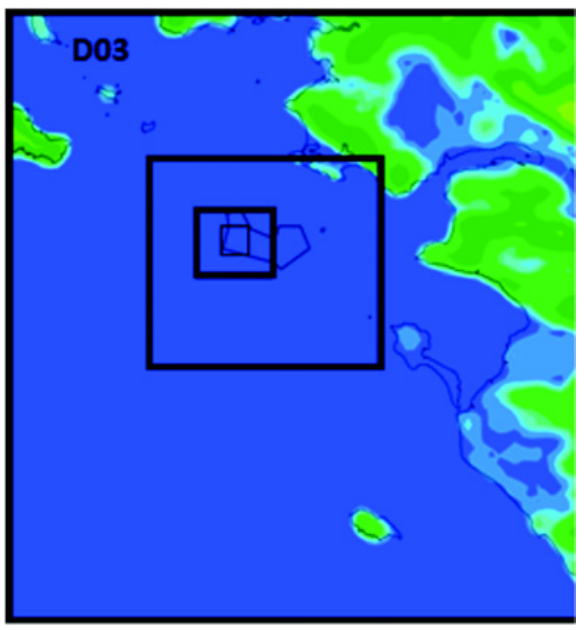

Figure 3. a) Scheme of grid nested domains, b) zoom to the 3 smallest domains.

equation model, the remaining use the WRF-LES equation model. The sub grid stress is modeled by a Smagorinsky model with a constant coefficient fixed to 0.18 [1]. The positions of the nested domains are illustrated on Figure 3.

The smallest domain measures $4 \mathrm{~km} \times 4 \mathrm{~km}$. The three dimensional wind field obtained in this simulation was then introduced as initial conditions in a micro scale model which uses full nonstationary LES calculations with SOWFA. During some tests it has emerged that the instantaneous boundary field from WRF could not be used as a boundary condition for SOWFA. The probable reasons are the lack of a relaxing zone and the incompatibility of the sub grid model.

\subsection{Wind regime selection results}

The wind resource assessment work was performed using time-series obtained in specific locations of the WRF simulation domain. A Python toolbox has been developed by INNOSEA to carry out full IEC 61400-1 standard wind result calculations. The outputs include a series of statistical analysis results. They include:

- Average wind speed at output heights

- Atmospheric density

- Turbulence intensity as a function of average wind speed and turbulence classes

- Wind direction distribution

- Wind speed statistical distribution (Weibull, Rayleigh, measured, specified) 

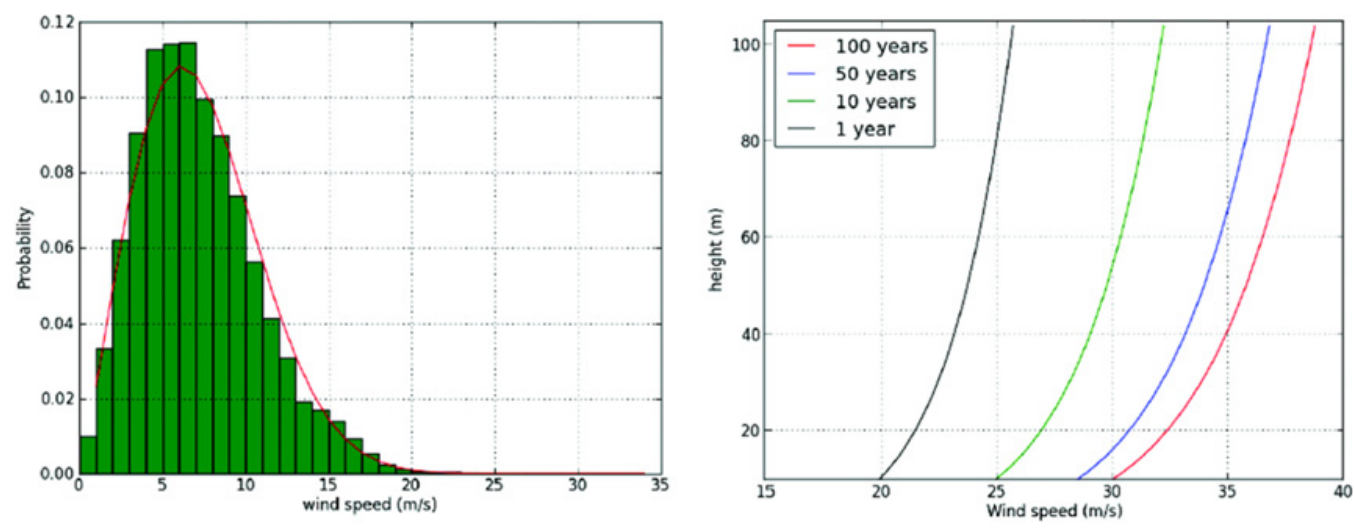

Figure 4. a) Wind speed Weibull distribution at turbine selected site, b) IEC $\mathrm{V}_{e 1}, \mathrm{~V}_{e 50} \& \mathrm{~V}_{e 100}$ extreme wind speed calculations.

- 50-year and 1-year Extreme wind speed at output locations

- Maximum speed for maintenance

- Model parameters and normal wind shear

- Wind profile model and its parameters

- Turbulence model and its parameters

- Model and parameters for extreme gusts 1-Y and 50-Y

- Extreme coherent gust model and parameters

- Extreme gust consistent with change of direction model and parameters

- Extreme wind shear model and parameters.

For the next steps of the work, the wind regime selection has lead to choose two cases:

- Average wind conditions with a wind speed at $10 \mathrm{~m}$ of $7.7 \mathrm{~m} / \mathrm{s}$ and a direction of $90^{\circ}$

- Severe wind conditions with a wind speed at $10 \mathrm{~m}$ of $28 \mathrm{~m} / \mathrm{s}$ and a direction of $235^{\circ}$.

\section{Wake effect simulation using large eddy simulation}

\subsection{Methodology overview}

The LES code SOWFA developed by NREL (National Renewable Energy Laboratory) and based on OpenFOAM was used to carry out simulations of flow around two wind turbines spaced $750 \mathrm{~m}$ (5.7 times the rotor diameter) one from another [5]. This spacing is considered as small and is similar to that used per example in Hornsrev I (Denmark). Detailed information about the method can be found in [3].

The work is divided in two simulation steps (Fig. 5):

- The first simulation generates the boundary layer without turbines by means of the ABL (Atmospheric Boundary Layer) solver.

- The second simulation uses the conditions just calculated but computes also wake effects using an actuator line model.

In the first simulation the atmospheric boundary layer is generated taking into account the results from WRF. In spite the fact that we run WRF simulation in LES mode, they produced a very homogeneous 
actuator line simulation

WRF result

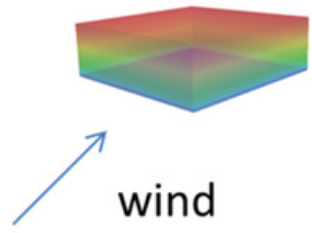

\section{.}
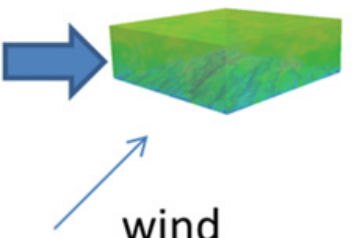

wind
$\mathrm{ABL}$ solver result
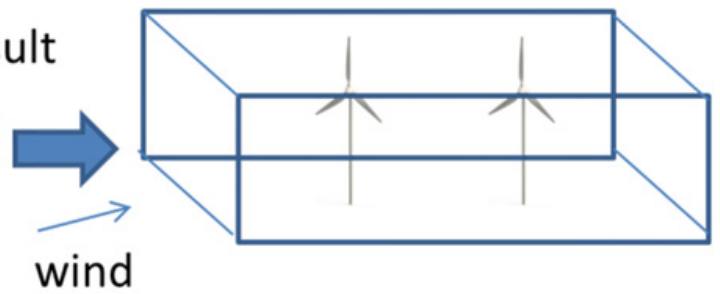

Figure 5. Scheme of the methodology.

wind field. So we decided to only use in the first step of the OpenFOAM simulations the wind field obtained with WRF. Moreover the flow was driven at the hub height by the mean speed and main direction of the WRF results. Periodic boundary conditions were then imposed in order to generate most realistic turbulent conditions. This simulation provides the flow characteristics of the domain, taking into account the turbulence caused by the ground roughness and that caused by the thermal conditions. Nevertheless we computed only neutral cases in this work (most neutral WRF periods were chosen). In the second simulation a LES with a wind turbine model (actuator line model) is carried out where the initialization of the flow field and the boundary condition were provided from the results of the first simulation. Here we obtain the influence of upwind turbine wakes on the turbulence to which a turbine is exposed.

\subsection{Numerical set-up}

The domain of the ABL solver measured $3 \mathrm{~km} \times 3 \mathrm{~km} \times 1 \mathrm{~km}$ in $\mathrm{x}$, $\mathrm{y}$ and $\mathrm{z}$-direction had a resolution of $20 \mathrm{~m} \times 20 \mathrm{~m} \times 20 \mathrm{~m}$ from the ground to $400 \mathrm{~m}$ and $40 \mathrm{~m} \times 40 \mathrm{~m} \times 40 \mathrm{~m}$ above. In a large-eddy simulation of a complex boundary layer with a horizontal resolution of $20 \mathrm{~m}$ turbulent properties could already be obtained with success by [6]. The time step was adjusted automatically. The initialization was done by a 3D field from WRF-LES results. We noticed that the development of turbulent structures was very poor and so decided to run the ABL solver $15000 \mathrm{~s}$ with periodic boundary conditions in order to get more realistic turbulence. We consider that the flow had achieved statistical stabilization at $12000 \mathrm{~s}$ that corresponds approximately to 6 times the eddy turn over time. The flow was driven by a constant horizontal mean wind speed at hub height. This forcing is done by adjusting the driving pressure gradient at each time step to correct the wind speed and direction continuously. For typical wind conditions we used wind speed of $7.7 \mathrm{~m} / \mathrm{s}$ and a direction of $90^{\circ}$ and for extreme ones $28 \mathrm{~m} / \mathrm{s}$ and a direction of $235^{\circ}$.

In the case of the simulation with actuator line model the domain had the same extent than in the first simulation but a maximal grid resolution of $3.75 \mathrm{~m} \times 3.75 \mathrm{~m} \times 5 \mathrm{~m}$ in proximity of the turbines and was stretched to a minimal grid resolution of $30 \mathrm{~m} \times 40 \mathrm{~m}$ in the upper part of the domain. The time step was fixed to $0.03 \mathrm{~s}$ in the case of typical wind conditions and to $0.015 \mathrm{~s}$ in the case of extreme ones. In the inflow direction the boundary conditions were provided from the ABL solver simulation, in the outflow direction an inlet-outlet condition was set. We did not consider neither the usual stop of the wind turbine at $25 \mathrm{~m} / \mathrm{s}$ nor the pitch adjusting that should normally be taken into account when wind speed exceeds. Therefor we analyzed the extreme case only poorly and focused the case of typical wind conditions for the turbulence statistics. The characteristics of the NREL $5 \mathrm{MW}$ turbine were used in both simulations. 
a)

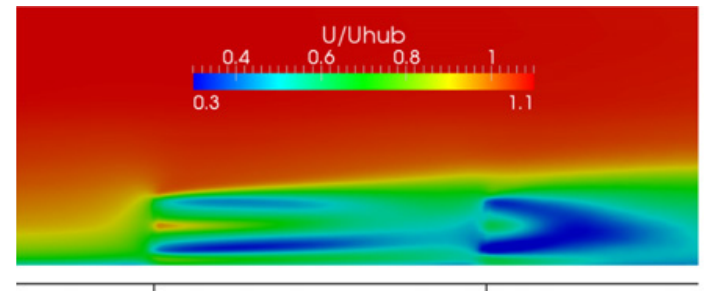

0

c)

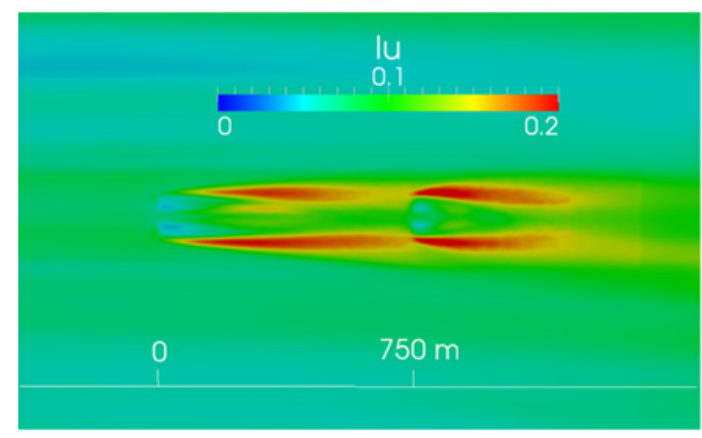

e)

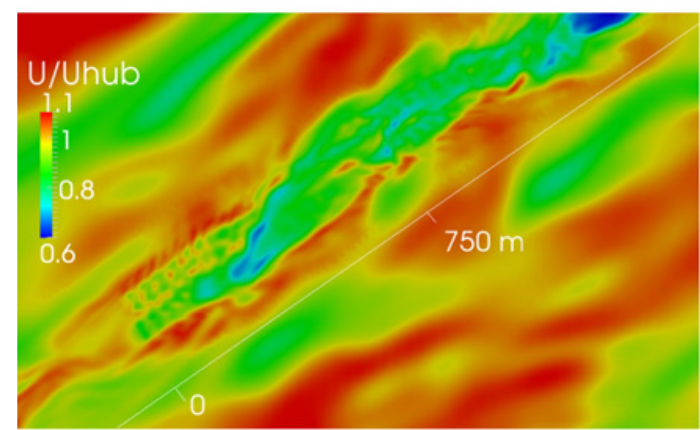

b)

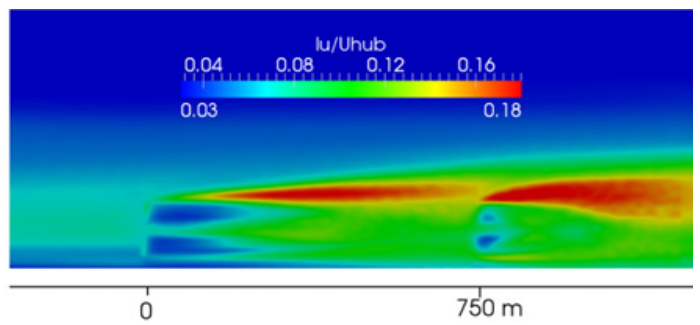

d)

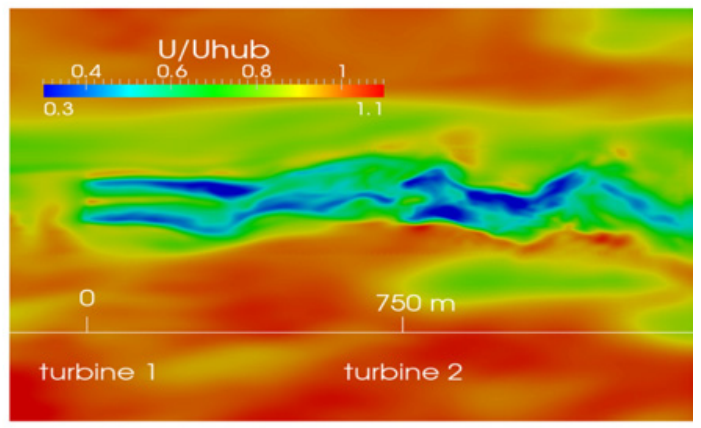

Figure 6. Horizontal plane (at $\mathrm{z}=90 \mathrm{~m}=$ Hhub) of a) normalized mean longitudinal velocity, b) longitudinal turbulent intensiy, c) Vertical plane of the turbulent intensity at the centre of the turbines, d) normalized instantaneous longitudinal velocity, (a) to d) concern typical wind conditions), e) normalized mean longitudinal velocity of extreme wind conditions.

\subsection{Mean characteristics of the turbulent flow behind the wind turbines}

Normalized mean and instantaneous wind characteristics of both simulations are shown in Figures 6a to e.

The region where the mean velocity is slowed down forms a thin continuous tunnel whose radius decreases slowly with downwind distance (Fig. 6a). At the downwind turbine we find a stronger decreasing of the mean velocity. The "tunnel's walls" are much thicker and its radius decreases much faster so that the zones of decreased velocity are completely joined at $3 \mathrm{~d}$ behind this second turbine. The same behavior is shown in [7] for a similar case. 
The maximal longitudinal turbulence intensity (Figs. $6 \mathrm{~b}$ and $6 \mathrm{c}$ ) at the tip top level is found at a downwind distance between 3 and $5 \mathrm{~d}$ behind the first turbine and at $3 \mathrm{~d}$ behind the second turbine. This observation is very well in agreement with [8]. We can also confirm that on a yz plane (not shown here) downwind the turbine the zone of maximum turbulence intensity form a "U". Nevertheless, we find slightly higher turbulence intensity at $\mathrm{z}=1.9 \mathrm{~d}$ than at the top tip level $(\mathrm{z}=1.4 \mathrm{~d})$. This can be explained by the ratio between the boundary layer and the wind turbines that is thicker in our case than in [8].

At typical wind conditions the tip speed ratio (TSR) is about 7.7. Looking at the instantaneous velocity (Fig. 6d) we find that the helical vortices caused by the tip vortices cannot be distinguished explicitly. They are driven downstream the turbine in a single shear layer (Blue colour on Fig. 6d). For the extreme wind conditions, with a TSR of 2.9, we are able to see explicit helical vortices formed by the blades when we represent instantaneous velocity (Fig. 6e). These have already been observed at a TSR of 5.7 by [7]. In our case they disappear behind the first turbine at half the distance between the two turbines and cannot reach the second turbine, event at this tip speed ratio. A more exhaustive study would be necessary to establish an exacte relation between TSR and the distance where these helical vortices disappear.

These observations are in agreement with previous LES [5, 7]. We will now investigate for this reason by means of higher momentum analyses. For these analyses we will focus on the period of typical wind conditions.

Turbulence intensity only provides us the proportion between the root mean square of the fluctuation and the velocity. In order to better understand the effect of the upwind turbine to the turbulent flow arriving at the downwind turbine we analyze here the spatial distribution of the turbulence by means of the skewness and the flatness defined by (8) and (9).

$$
\begin{aligned}
& \text { Skewness }=(8) \\
& \text { Flatness }=(9)
\end{aligned}
$$

The longitudinal skewness behind the first turbine in the simulation for typical wind conditions describes a regular pattern (Fig. 7a). Moreover we see in this case the formation of concentrical circles. The largest circle contains positive skewness that is located around the turbine's diameter. The alternation of negative and positive circles is clearly marked.

The alternation of negative and positive skewness circles signifies that the distribution of turbulent motions depends on its distance from the hub. The tip vortices reside between a distance of 100 and $140 \mathrm{~m}$ from the hub and are characterized by few strong fluctuations in the opposite direction of the flow and many weak ones in the flow direction. They contain for this raison negative longitudinal skewness.

The outermost concentrical circle is characterized by positive skewness and contains few strong positive fluctuations in flow direction and many weak ones in opposite direction. This is a consequence of the formation of the tip vortices. The same phenomenon, but much less intensive, appears also in the other side of the tip vortices, in the direction to the center of the turbine, so to form the third concentrical circle. Then, continuing towards the center, we find another circle with negative skewness (also less intensive than the outer one) which corresponds to the zone where the flow describes the hub vortices. The position of hub and tip vortices in the yz plane 1 rotor diameter downwind to the turbine are similar to those pointed out in the analysis of the vorticity carried out by [8]. Next to the zone of this second vortex zone we find the inner zone of the serie of concentrical circles, where we find weak positive longitudinal skewness again.

Hence strong negative skewness indicates in this yz plane the zones where a vortex is formed and strong positive skew is found in their neighbourhood zones.

The spatial evolution of skewness is directly linked to that of flatness (Fig. 7b). While skewness tells us if negative fluctuations or positive fluctuations dominate in contribution, flatness indicates us if fluctuations are homogeneous or have very different amplitudes. We see in Figures $7 \mathrm{~b}, \mathrm{~d}$ and $\mathrm{f}$ that the 
a)

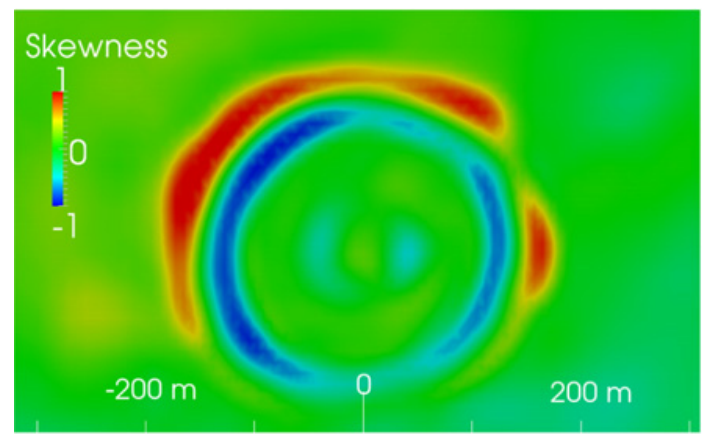

c)
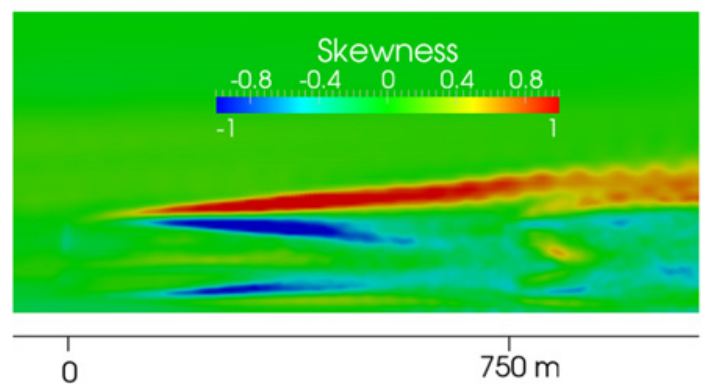

e)

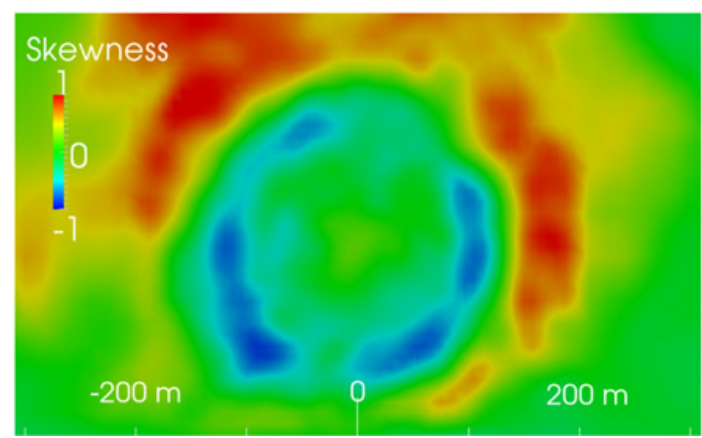

b)

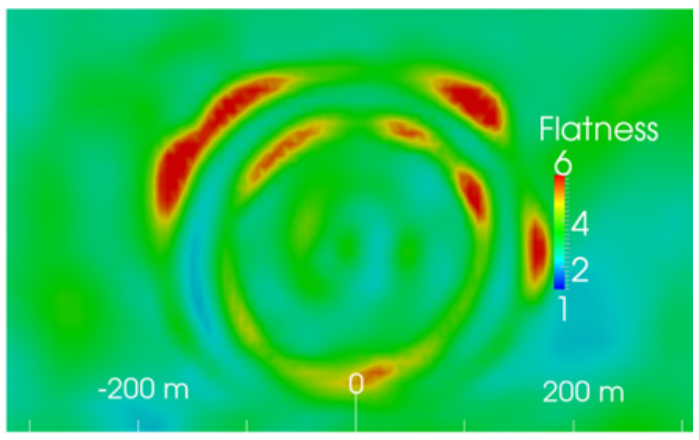

d)

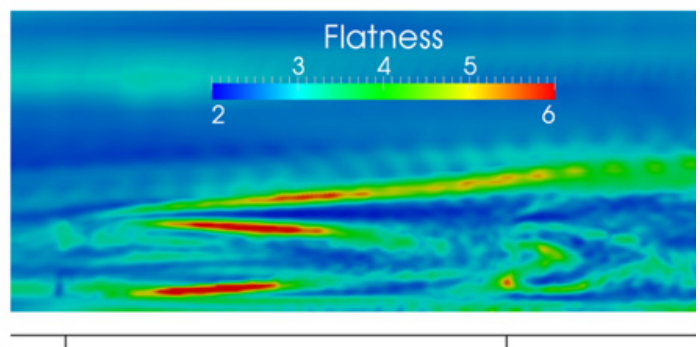

0

$750 \mathrm{~m}$

f)

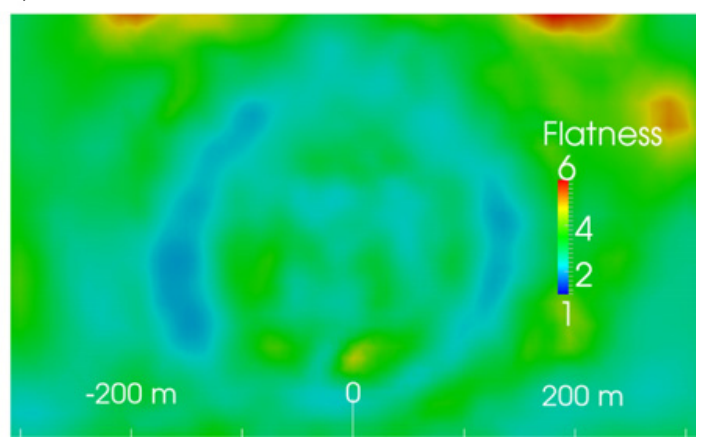

Figure 7. a) yz plane of the longitudinal skewness behind the first turbine at $x / D=1, b)$ yz plane of the longitudinal flatness behind the first turbine at $x / D=1, c) x z$ plane of the longitudinal skewness, d) $x z$ plane of the longitudinal flatness, e) yz plane of the longitudinal skewness behind the second turbine at $\mathrm{x} / \mathrm{D}=1, \mathrm{f}$ ) $\mathrm{yz}$ plane of the longitudinal flatness behind the second turbine at $\mathrm{x} / \mathrm{D}=1$. This figure shows only results for typical wind conditions.

flatness is important when large positive or large negative skewness appear, so in the zones of tip and hub vortices but also around the circular zone of tip vortices.

The flow pattern described here above is found when the upstream flow is not already perturbed by other turbines. One of the question that we will answer in this work is : What is the distribution of turbulence that is transported to the downwind turbine?

When we look at the downwind turbine, we see that the flow field is strongly influenced by the upwind turbine. The evolution of the fluctuation's pattern can be observed in the horizontal plane of 
skewness and flatness (Figs. 7c and d). Again the evolution of flatness is linked to that of skewness and we observe a decreasing of both at about $\mathrm{x}=3 \mathrm{~d}$. Concentrical circles are still observed behind the second turbine in both cases (Figs. 7e and f), but the pattern is much less regular than in the case of the first turbine. Interestingly both, skewness and flatness are considerably lower behind the second turbine than behind the first one. While turbulence intensity increases after the second turbine, the distribution of this turbulence is distributed in a more homogeneous way.

\section{Conclusion and discussions}

An assessment study by means of the WRF meso-scale model allowed to obtain the wind conditions over 12 years on the site of the future offshore wind farm of Saint Nazaire. A comparison with measurements from a nearshore mast allowed obtaining a mean error in wind velocity less than 5\%. After a statistical study, a regime of extreme wind conditions and one of average wind speeds encountered offshore were chosen and ran in LES mode with WRF that is a very recent option of this tool. The results were then used with SOWFA, a Large Eddy Simulation tool based on OpenFOAM and developed by the NREL. SOWFA provides non-stationary wind velocity outputs which lead to study wake effects by means of an Actuator Line model. Two turbines were placed in the turbulent flow and turbulent statistics were analyzed and discussed. Mean velocity and turbulence intensities were in agreement with results from literature. Moreover the flatness and the skewness of the distribution of the fluctuations in the wake were investigated and it was shown that, while turbulence intensity increases after the second turbine, the distribution of this turbulence is distributed in a more homogeneous way.

To merge the gap between meso-scale and micro-scale modelling, the next step should be the development of a grid nesting between WRF and SOWFA taking into account not only initial conditions but instantaneous boundary conditions.

In the case of statistical analyses INNOSEA aims to link statistical parameters like skewness and flatness with production and fatigue of turbine characteristics and components. These two last are often only estimated in function of velocity and turbulence intensity.

\section{References}

[1] Talbot C, Bou-Zeid E, Smith JA (2012), Nested mesoscale-large eddy simulations with WRF: performance in real test cases" The Journal of Hydrometeorology

[2] Wyngaard JC 2004, Towards numerical modelling in the "Terra incognita", J. Atmos. Sci. 61: $1816-1826$

[3] Churchfield MJ, Lee S, Michalakes J, Moriarty PJ (2012), A numerical study of the effect of atmospheric and wake turbulence on wind turbine dynamics, Journal of Turbulence 13

[4] Dudhia J (2011). Overview of WRF Physics. Technical report, National Center for Atmospheric Research

[5] NWTC Design Codes (SOWFA by Matt Churchfield and Sang Lee). http://wind.nrel.gov/designcodes/simulators/SOWFA/

[6] Maché M (2012), Représentation multi-échelle des transferts entre couhce de canopée urbaine et atmosphere à l'échelle de la ville, phd-thesis, Ecole Centrale de Nantes, France, $196 \mathrm{p}$

[7] Storey RC, Norris SE, Stol KA, Cater JA (2011), Coupled Large Eddy Simulation of Dynamically Controlled Wind Turbines, EWEA Offshore 2011, Proceedings

[8] Wu YT, Porté-Agel F (2013), Simulation of Turbulent Flow Inside and Above Wind Farms: Model Validation and Layout Effects, Boundary-Layer Meteorolgy 146: 181-205

[9] Zhang W, Markfort CD, Porté-Agel F (2012), Near-wake flow structure downwind of a wind turbine in a turbulent boundary layer, Exp Fluids 52: 1219-1235 